\title{
Publisher's Note: Enhanced optical activity in hyperbolic metasurfaces \\ [Phys. Rev. B 96, 235403 (2017)]
}

O. V. Kotov and Yu. E. Lozovik

(Received 15 December 2017; published 26 December 2017)

DOI: 10.1103/PhysRevB.96.239905

This paper was published online on 4 December 2017 with an omission of an author affiliation and an error in Fig. 3. The author affiliations should read as:

O. V. Kotov ${ }^{1,2, *}$ and Yu. E. Lozovik ${ }^{1,2,3,4, \dagger}$

${ }^{1}$ N. L. Dukhov Research Institute of Automatics (VNIIA), 127055 Moscow, Russia

${ }^{2}$ Institute of Microelectronics Technology and High Purity Materials, Russian Academy of Sciences, 142432 Chernogolovka, Russia

${ }^{3}$ Institute for Spectroscopy, Russian Academy of Sciences, 142190 Troitsk, Moscow, Russia

${ }^{4}$ National Research University Higher School of Economics, 101000 Moscow, Russia

Figure 3 has been replaced and the author affiliations have been corrected as of 8 December 2017. The author affiliations and figure are correct in the printed version of the journal. 